\title{
Effectiveness of Integration of Mindfulness -Based Cognitive Therapy and Recovery Cognitive Behavioral Therapy on Adolescents with Spectrum Bipolar Disorder
}

\author{
Hajar Hasani ardekani*, Hojaolah Javidi, Amir Hooshang Mehryar, Ebrahim Hoseni \\ Department of Psychology, faculty of education and psychology, Marvdasht branch, Islamic Azad University, \\ marvdasht, Iran
}

*Corresponding Author: Dr. Hajar Hasani ardekani, Department of Psychology, faculty of education and psychology, Marvdasht branch, Islamic Azad University, marvdasht, Iran, Tel: +989178855899; E-mail: aminalvani64@gmail.com

Received: 24 August 2018; Accepted: 31 August 2018; Published: 06 September 2018

\begin{abstract}
Objective: The aim of this study was Effectiveness of Integration of Mindfulness -based cognitive therapy (MBCT) and recovery cognitive behavioral therapy (RCBT) on adolescents with spectrum bipolar disorder (BD).

Method: BD diagnosis in 80 adolescents was based on DSM-IV TR that patients were randomly assigned to one of the following: Experimental group under combined treatment MBCT \& RFCBT, and groups MBCT, RFCBT and Control group (TAU) under pharmacological treatment. The questionnaires used in the research included: Manian Yang MYR questionnaire, K-SADS quality of life questionnaire, impulsivity questionnaire, bipolar depression questionnaire, Beck anxiety inventory, Coke drug abuse questionnaire. We used an analysis of variance (MANOVA), including one or two factors, with repeated measures at different evaluation times: baseline, posttreatment, 6-month follow-up.
\end{abstract}

Results: We found significant between-group differences at all evaluation times after the treatment. The experimental group showed that the effect of MBCT treatment on improving the psychiatric and psychological symptoms of individuals in three stages (pre-test, post-test and follow-up) (849/0) is significant. The effect of RFCBT treatment on improving the psychiatric and psychological symptoms of individuals in three stages (pre-test, post-test and follow-up) is somewhat significant and the effect of treatment (group membership) in the post-test and follow-up stage $0.27 \%$ and $0.31 \%$, respectively, meaning that $0.27 \%$ of individual differences in the improvement of psychiatric and psychiatric symptoms (during the test) and 31/0 percent (follow-up) for differences in membership 
group (treatment effect) and finally the integration of psychiatric and psychological MBCT and RFCBT improves the symptoms of spectrum bipolar disorders exist.

Conclusions: Our results suggest that a combined treatment is effective in patients with refractory bipolar disorder. Suggestions for future research are commented on.

Keywords: Mindfulness -based cognitive therapy; Recovery cognitive behavioral therapy; Bipolar disorder

\section{Introduction}

Bipolar disorder (BD) involving a condition of multiple aspects an individual's life. It is one of the most disabling illnesses globally, being the 18th leading reason of disability, generally because of its deleterious effects on social and health functioning compared with other mental disorder [1,2]. There is much disagreement about where to draw the boundaries of the bipolar spectrum, but some investigators include subsyndromal manic episodes, manic or hypomanic episodes triggered by antidepressants, or agitated depression [3, 4]. In spite of an appropriate treatment with mood stabilizers.in addition to, even without presentation of rapid cycling, these patients may suffer frequent relapses and have severe difficulties in their social-occupational functioning. All this is connected with elevated healthcare costs [5]. In its various forms bipolar disorder affects approximately 5.7 million Americans [6]. Ninetyeight percent of individuals with bipolar disorder have another lifetime psychiatric disorder that warrants treatment [7]. Psychosocial treatment researchers felt that if they could work with people with bipolar disorder to learn more about their illness, monitor symptoms, help them to become more adherent, and adjust their behavior and their environment in ways that would minimize risk factors (irregular medication adherence, lack of sleep, alcohol and substance abuse, family criticism, stress) and maximize protective factors (regular schedules, etc.), that this would have beneficial effects on the course of the illness and ultimately improve people's quality of life [8]. Mindfulnessbased cognitive therapy (MBCT) is a depressive relapse prevention program that combines aspects of cognitive therapy and training in mindfulness (meditation). Specifically developed to prevent unipolar depressive relapse [9] MBCT enables people to become more aware of their thoughts without judgment and to viewing them as passing mentalevents [10].

In individuals with three or more depressive episodes, MBCT allowed reducing the risk of relapse by half [11-13] and delayed the time to depressive relapse [13]. It was more effective than maintenance antidepressant treatment in reducing residual depressive symptoms and psychiatric comorbidity, and in improving quality of life among patients with recurrent depression, in full or partial remission at inclusion [14]. Preliminary results suggested that MBCT might be beneficial in patients with active symptoms of depression as well [15-17]. In most original MBCT trials, bipolar disorder was an exclusion criterion [11, 14]. Therefore, little is known about its efficacy in individuals with bipolar disorder. Recent studies [10, 18, 19] included some bipolar patients (type I and II) in samples of depressed unipolar individuals and concluded that MBCT allowed reducing depressive symptom severity. When focused on between-episode anxiety and depressive symptoms in unipolar and bipolar patients with suicidal ideation or behavior [10, 19]. Psychoeducational programs and cognitive-behavioral techniques (CBT) are, to date, the patientfocused approaches that have shown clear evidence of efficacy in randomized studies. It is also worthwhile to note 
the strong evidence that exists in support of the use of family-focused approaches [20, 21] CBT may be efficacious for treating postmanic `downs` [22]. After a hypomanic or manic phase, the patient may appear hypoactive and abulic. In such cases, antidepressant treatment should probably be avoided because there may be a risk of inducing a switch into mania or rapid cycling [23]. Self-control techniques, stress management and inoculation, exposition and coping strategies might be useful in the treatment of specific problems caused by the illness.

Some open-series studies and case reports suggested that CBT may be useful as a coadjunctive treatment for rapidcycling patients [24]. To date, psychological treatments have utilized an array of interventions. These include psychoeducation about bipolar disorder, mood monitoring and relapse prevention, communication and problemsolving training, cognitive restructuring (a technique to challenge maladaptive thoughts), activity scheduling, social rhythm adjustments, and interpersonal techniques. These have been combined in various forms in individual, group, or family treatments that were implemented at different stages of this illness (acutely ill, stable, etc.). Except Integration of Mindfulness - based cognitive therapy and recovery cognitive behavioral therapy Therefore, the purpose of this research but to date research in the field Effectiveness of Integration of Mindfulness -based cognitive therapy and recovery cognitive behavioral therapy on adolescents with spectrum bipolar disorder not accepted.

\section{Methods}

\subsection{Subjects}

The study was conducted in a Psychiatric Hospital Professor Mohariri Shiraz in the treatment of bipolar disorders, within the Department of Psychiatry of the Shiraz University Hospitals. The study protocol was approved by the Ethics Committee of the Department of Psychiatry and each subject gave written informed consent. Inclusion criteria were: age more than18; no hospitalization for psychiatric conditions during the last 3 months; severity of hypomanic symptoms at inclusion less than 8 on the Young Mania Rating Scale (YMRS) [25, 26]. There was no cut-off score on the MADRS although patients with a score inferior or equal to 15 at time of inclusion were preferentially included [27]. Regular substances abuse was an exclusion criteria. In keeping with naturalistic clinical conditions, recruitment was not restricted to patients in full remission, nor to individuals without pharmacological treatment. Among 80 patients included in the study, one did not complete the initial assessment nor attend the first session and six dropped-out after attending less than four sessions, the minimal effective dose of MBCT in accordance with previous trials [11-13].

\subsection{Procedure}

This study is a randomized clinical trial design. Among the cases of bipolar patients referring to the psychiatric clinic professor Moharri Shiraz during the last year, there were 200 patients. 80 bipolar patients are selected as available. After obtaining informed consent, patients are randomly divided into 4 groups. The first group receives the combination of the two treatments (MBCT \& RFCBT), the second group is under treatment (RFCB), the third group receives treatment (MBCT) and the other group is controlled (TAU). All groups use medication and then they are evaluated using the tools listed. In the TAU group, the treatment is not performed and after the other groups' treatment, the questionnaires are again answered by the patients in the control group. The questionnaires used in the 
research included: Manian Yang MYR questionnaire, K-SADS quality of life questionnaire, impulsivity questionnaire, bipolar depression questionnaire, Beck anxiety inventory, Coke drug abuse questionnaire.

\subsection{Intervention}

Group 1: Combined Therapy (MBCT \& RFCBT): Protocols based on the scheme, in which MBCT treatment is merged with classic CBT therapy and recovery therapy which includes 1.5 -hour sessions, in which formulation and CBT therapy techniques plus meditation exercises focus on recovery.

Group 2: Cognitive behavioral therapy focused on the recovery of (RFCBT), the structure and formulation of treatment based on the treatment protocol (Basco Vrache, 2005) and (Jones, 2012).

Group 3: Cognitive-focused therapy focused on mind awareness (MBCT), the structure and formulation of treatment based on the treatment protocol (Decker, 2012).

Group 4: Typical Treatment (TAU), the Patients in this group continue their treatment without any intervention.

\subsection{Data analysis}

Analyses were conducted on the 80 participants who attended at least eight sessions, for all four treatment groups MBCT, Integration MBCT \& RFCBT, RFCBT and TAU prevalent treatment, Frequency tables were used to describe perceived benefit from the program, perceived usefulness of the program ingredients. Preand post-MBCT, Integration MBCT \& RFCBT, RFCBT and TAU prevalent treatment, assessments were described with median (range) and compared using MANOVA signed ranks tests. Associations were tested with Spearman rank-order correlation coefficients (rs). Statistical significance was set at 0.05 (two-tailed tests). Data analysis was performed using SPSS 16 (SPSS Inc., Chicago, IL).

\section{Results}

To evaluate the treatment of MBCT, improvement of psychiatric and psychological symptoms of bipolar disorder is used through repeated measures of variance analysis. In which the effect of MBCT treatment improves psychiatric and psychological symptoms in the first test group in three stages of pre-test, post-test and follow-up. The results are presented in the following tables 1 and 2 .

\begin{tabular}{|l|l|l|l|l|l|}
\hline Multivariate test & Value & F & DF & Sig. & Partial Eta Squared \\
\hline Pillai's trace & 0.849 & $36.509^{\mathrm{a}}$ & 13 & 0.001 & 0.849 \\
\hline Wilkes's lambda & 0.151 & $36.509^{\mathrm{a}}$ & 13 & 0.001 & 0.849 \\
\hline Hoteling's trace & 5.617 & $36.509^{\mathrm{a}}$ & 13 & 0.001 & 0.849 \\
\hline Roy's largest root & 5.617 & $36.509^{\mathrm{a}}$ & 13 & 0.001 & 0.849 \\
\hline
\end{tabular}

${ }^{a}$ Modified for Multiple Comparison: Bonferron.

Table 1: The results of repeated variance analysis of the MBCT treatment group for the variable of psychological and psychological symptoms improvement. 


\begin{tabular}{|l|l|l|l|l|l|l|}
\hline \multirow{2}{*}{ Levels } & \multirow{2}{*}{ Difference of meanings } & Standard error & \multirow{2}{*}{ P } & \multicolumn{2}{|l|}{ Confidence interval } \\
\cline { 5 - 7 } & & & Min & Max \\
\hline \multirow{3}{*}{ Pre } & post & $4.387^{*}$ & $0 / 909$ & 0.001 & 1.917 & 6.856 \\
\cline { 2 - 7 } & Follow up & $4.433^{*}$ & $0 / 543$ & 0.001 & 2.959 & 5.908 \\
\hline \multirow{3}{*}{ Post } & pre & $-4.387^{*}$ & $0 / 909$ & 0.001 & -6.856 & -1.917 \\
\cline { 2 - 7 } & Follow up & 0.047 & $0 / 551$ & 1 & -1.451 & 1.545 \\
\hline \multirow{2}{*}{ Follow up } & pre & $-4.433^{*}$ & $0 / 543$ & 0.001 & -5.908 & -2.959 \\
\cline { 2 - 7 } & Post & 0.047 & $0 / 551$ & 1 & 1.451 & -1.451 \\
\hline
\end{tabular}

Table 2: Bonferron post hoc test results analysis of variance of repeated measures of MBCT treatment group for improvement of psychiatric and psychological symptoms.

The results of Table 1 show that all tests have meaningful significance less than 0.01 . The Wilcox lambda test, which is more powerful than other tests, with a value of (0.151) and (36.509) F and 0.849, the separation of EAs is significant at a significant level of 0.01 . The effect of treatment of 0.849 with a potency of 1 that indicates the adequacy of the sample size for the conclusion suggests that the effect of MBCT treatment on improving the psychiatric and psychological symptoms of individuals in three stages (pre-test, post-test and follow-up) (0.849) is significant. In other words, there is a significant difference between the mean improvement in psychiatric and psychological symptoms of this group in three stages. Consequently, the research hypothesis is not rejected. To evaluate the difference between the meanings, Bonferron's post hoc test was used. The results of Table 2 show that between the improvement of psychiatric and psychological symptoms in the post-test (92.22) and pre-test (96.71), pre Test (71.96) and follow-up (92.28) have a significant difference. However, there was no significant difference between the mean of improvement of psychiatric and psychological symptoms in follow up and post-test stages. To investigate the treatment of RFCBT, psychological and psychological symptoms of bipolar disorder have been utilized through repeated measures of variance analysis. In which the effect of the RFCBT treatment method is compared in three stages: pre-test, post-test and follow-up. The results are presented in the following tables.

\begin{tabular}{|l|l|l|l|l|l|}
\hline Multivariate test & Value & F & DF & Sig. & Partial Eta Squared \\
\hline Pillai's trace & 0.494 & $6.355^{\mathrm{a}}$ & 13 & 0.012 & 0.494 \\
\hline Wilkes's lambda & 0.506 & $6.355^{\mathrm{a}}$ & 13 & 0.012 & 0.494 \\
\hline Hoteling's trace & 0.978 & $6.355^{\mathrm{a}}$ & 13 & 0.012 & 0.494 \\
\hline Roy's largest root & 0.978 & $6.355^{\mathrm{a}}$ & 13 & 0.012 & 0.494 \\
\hline
\end{tabular}

${ }^{a}$ Modified for Multiple Comparison: Bonferron.

Table 3: The results of repeated measures analysis of variance of the RFCBT treatment group for the variable of psychological and psychological symptoms improvement. 


\begin{tabular}{|l|l|l|l|l|l|l|}
\hline \multirow{2}{*}{ Levels } & \multirow{2}{*}{ Difference of meanings } & \multirow{2}{*}{ Standard error } & \multirow{2}{*}{ P } & \multicolumn{2}{|l|}{ Confidence interval } \\
\cline { 5 - 7 } & & & Min & Max \\
\hline \multirow{2}{*}{ Pre } & post & $2.490^{*}$ & 0.677 & 0.007 & 0.649 & 4.331 \\
\cline { 2 - 7 } & Follow up & $4.357^{*}$ & 0.660 & 0.009 & 0.563 & 4.150 \\
\hline \multirow{2}{*}{ Post } & pre & $-2.490^{*}$ & 0.677 & 0.007 & -4.331 & 0.649 \\
\cline { 2 - 7 } & Follow up & -0.133 & 0.252 & 1 & 0.817 & 0.550 \\
\hline \multirow{2}{*}{ Follow up } & pre & $-2.357^{*}$ & 0.660 & 0.009 & -4.150 & 0.563 \\
\cline { 2 - 7 } & Post & 0.133 & 0.252 & 1 & 0.550 & 0.817 \\
\hline
\end{tabular}

Table 4: Bonferron post hoc test results Analysis of variance of repeated measures of RFCBT treatment group for improvement of psychiatric and psychological symptoms.

The findings of Table 3 indicate that all tests have meaningful significance less than 0.05 . The Wilcox lambda test, which is more powerful than other tests, with a value of (0.506) and (6.355) $\mathrm{F}$ and 0/494, the separation of EAs is significant at a significant level of 0.05 .The effect of treatment of 0.449 with a potential of 0.815 which indicates the adequacy of the sample size for the conclusion suggests that the treatment method did not have a significant effect. However, it can be said that the effect of RFCBT treatment on improving the psychiatric and psychological symptoms of individuals in three stages (pre-test, post-test and follow-up) is somewhat significant. In other words, there is a significant difference between the mean improvement in psychiatric and psychological symptoms of this group in three stages. Consequently, the research hypothesis is not rejected. To evaluate the difference in mean, Bonferron's post hoc test was used. The results of Table 4 show that between the improvement of psychiatric and psychological symptoms in the post-test (93.59\%) and pre-test (96.83\%), pre-test (083.96) and follow-up (93.72) there is a significant difference but there was no significant difference between the mean improvement of psychiatric and psychological symptoms in the follow up and post-test stages. As a result, the effect of therapeutic intervention is stable over time. Covariance analysis was used to investigate the integration of MBCT and RFCBT in improving psychiatric and psychological symptoms of bipolar disorder.

\begin{tabular}{|l|l|l|l|l|l|l|}
\hline \multirow{2}{*}{ Source of change } & Levels & Average squares & DF & F & Sig. & Partial Eta Squared \\
\hline \multirow{2}{*}{ pre (C) } & Post & 594.10013 & 1 & 824.126 & 0.001 & 0.969 \\
\cline { 2 - 7 } & Follow up & 418.10328 & 1 & 628.21 & 0.001 & 0.981 \\
\hline \multirow{2}{*}{ Group } & Post & 900.59 & 2 & 7.566 & 0.002 & 0.27 \\
\cline { 2 - 7 } & Follow up & 741.46 & 2 & 9.497 & 0.001 & 0.317 \\
\hline
\end{tabular}

Table 5: The results of covariance analysis of the effect of integration of MBCT and RFCBT improve psychiatric and psychological symptoms of bipolar spectrum disorder in two stages of post-test and follow-up with considering the pre-test phase as the quarantine variable.

The findings of Table 5 show that although the effect of quart variable (pre-test) in post-test with (644.824) $\mathrm{F}$ and follow-up with (F2898) is F and the level of significance is less than 0.001 is meaningful, in the source of change, 
there is a significant difference between the means of improving the psychiatric and psychological symptoms in the target group in the post-test and follow up stages. The effect of treatment (group membership) in the post-test and follow-up stage was $0.27 \%$ and $0.31 \%$ respectively, meaning that $0.27 \%$ of the individual differences in the improvement of psychiatric and psychiatric symptoms (in the post-test) and $0.31 \%$ (follow-up) due to differences in group membership (treatment effect). The statistical power of 0.972 and 0.929 in the two groups indicates the adequacy of the sample size for the conclusion. Bonferron's post hoc test was used to examine the difference between the means. The results are presented in the following table.

\begin{tabular}{|c|c|c|c|c|c|c|c|}
\hline \multirow{2}{*}{ Levels } & \multirow{2}{*}{ Group (I) } & \multirow{2}{*}{ Group (J) } & \multirow{2}{*}{$\begin{array}{l}\text { Difference } \\
\text { of meanings }\end{array}$} & \multirow{2}{*}{$\begin{array}{l}\text { standard } \\
\text { error }\end{array}$} & \multirow{2}{*}{ Sig } & \multicolumn{2}{|c|}{ Confidence interval } \\
\hline & & & & & & Min & Max \\
\hline \multirow{8}{*}{ post } & \multirow{2}{*}{ MBCT } & RFCBT & -1.899 & 1.028 & 0.215 & 4.464 & 0.666 \\
\hline & & MBCT, RFCBT & $-4^{*}$ & 1.029 & 0.001 & -6.567 & -1.432 \\
\hline & \multirow{2}{*}{ RFCBT } & MBCT & 1.899 & 1.028 & 0.215 & -0.666 & 4.464 \\
\hline & & MBCT, RFCBT & -2.101 & 1.03 & 0.143 & -4.567 & 0.469 \\
\hline & \multirow{2}{*}{$\begin{array}{l}\text { MBCT, } \\
\text { RFCBT }\end{array}$} & MBCT & $4^{*}$ & 1.029 & 0.001 & 1.432 & 4.567 \\
\hline & & RFCBT & 2.101 & 1.03 & 0.143 & -0.469 & 4.671 \\
\hline & \multirow{2}{*}{ TAU } & MBCT & $4^{*}$ & 1.029 & 0.001 & 1.432 & 4.567 \\
\hline & & RFCBT & $2 / 101$ & 1.03 & 0.143 & $0.469-$ & 4.671 \\
\hline \multirow{8}{*}{ Follow up } & \multirow{2}{*}{ MBCT } & RFCBT & $-2.089^{*}$ & 0.81 & 0.041 & -4.111 & -0.067 \\
\hline & & MBCT, RFCBT & $3.513-$ & 0.811 & 0.001 & -5.537 & -1.488 \\
\hline & \multirow{2}{*}{ RFCBT } & MBCT & $-2.089 *$ & 0.81 & 0.041 & -0.067 & 4.111 \\
\hline & & MBCT, RFCBT & -1.424 & 0.812 & 0.261 & -3.45 & 0.603 \\
\hline & \multirow{2}{*}{$\begin{array}{l}\text { MBCT, } \\
\text { RFCBT }\end{array}$} & $\mathrm{MBCT}$ & $-3.513 *$ & 0.811 & 0.001 & 1.488 & 5.375 \\
\hline & & RFCBT & 1.424 & 0.812 & 0.261 & -0.603 & 3.45 \\
\hline & \multirow{2}{*}{ TAU } & MBCT & $4 *$ & 1.029 & 0.001 & 1.432 & 4.567 \\
\hline & & RFCBT & 2.101 & 1.03 & 0.143 & -0.469 & 4.671 \\
\hline
\end{tabular}

Table 6: The results of Bonferron post hoc test for the effectiveness of MBCT and RFCBT integration on the improvement of psychiatric and psychological symptoms.

Regarding the results of Table 1, it can be said that in the post-test phase, only a significant difference was observed between the mean improvement of the psychiatric and psychological symptoms of the MBCT group and the integration of MBCT and RFCBT. Here, the mean and improvement of psychiatric and psychological symptoms of MBCT individuals is lower than that of the MBCT and RFCBT integration group. However, in the follow up phase, there is a significant difference between the MBCB and RFCBT treatment groups and the integration of MBCT and RFCBT, which means that the mean improvement in the psychiatric and psychological symptoms of the MBCT group is lower in both groups.

\section{Discussion}

The results of this study show that between the improvement of psychiatric and psychological symptoms in the posttest and pre-test, pre Test and follow-up have a significant difference. However, there was no significant difference 
between the mean of improvement of psychiatric and psychological symptoms in follow up and post-test stages (table1). Strawn Jeffrey and et al,(2016) showed that MBCT-C treatment in anxious youth with a familial history of bipolar disorder is associated with increased activation of brain structures that sub serve interception and the processing of internal stimuli-functions that are ostensibly improved by this treatment. The results of Table 2 show that between the improvement of psychiatric and psychological symptoms in the post-test $(93.59 \%)$ and pre-test (96.83\%), pre-test (083.96) and follow-up (93.72) there is a significant difference but there was no significant difference between the mean improvement of psychiatric and psychological symptoms in the follow up and post-test stages. As a result, the effect of therapeutic intervention is stable over time. Covariance analysis was used to investigate the integration of MBCT and RFCBT in improving psychiatric and psychological symptoms of bipolar disorder. Isaaci, Chobrava, Limina and Gonzalez Pinto (2010), in their research entitled what is the method for a psychological intervention program for patients with refractory bipolar disorder? It was concluded that the experimental group had less hospitalization in comparison with the control group, and there was a significant difference in depression and anxiety in relation to mania and inappropriate treatment after treatment. Analysis of intra-group differences An experiment showed a decrease in mania and depression, anxiety and incompatibility, while in the control group, the number of admissions and the high level of mania increased anxiety and incompatibility. Finally, it can be said that the reason that some patients do not respond to treatment is that there is an explanation that may be related to the defect in intervention, in that the techniques and training used to reduce these symptoms are inadequate or disproportionate have been. And combined therapy has a better performance. Finally, our results suggest that suggestions for future combinative therapies are appropriate for patients with bipolar disorder resistant. And other therapies are combined and compared.

Gart et al. (2015) used MBCT for adolescents with anxiety disorders susceptible to bipolar disorder. This treatment increased attention adjustment, awareness and decreased anxiety. A study in 2016 was conducted by DE Place and colleagues on psychological problems with adolescents with behavioral problems. Group therapy was performed based on cognitive-mindedness. That adolescents have recovered symptoms of depression, rumination, emotional regulation, and impulsivity. On the other hand, bipartisan teenagers have a bad mood (Agasson 2011, quoted by Garth 2015) as a result of the increased activity of the brain membrane called PLPFCE which causes an emotional maladaptation the increase in PLPFCE is associated with mania symptoms. Considering that the MBCT is involved in emotional regulation, it may be effective for bipolar teenagers who have emotional maladaptation. Mindfulness and attention are awareness of the current experience (Baron 2008). Patients with bipolar disorder usually do not have insight, they must be aware of their excitement with cognitive behavioral therapy. So that they can be involved in a vigorous participation in the transcending process of living. In general, the integration of psychological treatments and timely treatment will prevent the complications of this severe and fatal disease.

The present study was one of the initial attempts in Iran, to check the feasibility and Integration of Mindfulness based cognitive therapy and recovery cognitive behavioral therapy for Bipolar disorder. While interpreting the findings, it should be noted that Integration of Mindfulness - based cognitive therapy and recovery cognitive behavioral therapy was found to be suitable for clients in partial remission, with relatively fewer episodes and residual symptoms. Small sample size is the significant limitation of the present study; these findings have restricted 
generalizability. In addition, there was absence of a control group to compare the significance of therapeutic gains. We suggest future studies should aim for larger sample sizes to allow for rigorous statistical analysis and longer follow-up assessments to confirm these preliminary findings.

\section{Acknowledgment}

We thank Amin Alvani for her expert technical support in the preparation of this manuscript.

\section{References}

1. Vos T, Flaxman AD, Naghavi M, et al. Years lived with disability (YLDs) for 1160 sequelae of 289 diseases and injuries 1990-2010: a systematic analysis for the Global Burden of Disease Study 2010. Lancet 380 (2012): 2163-2196.

2. Sanchez-Moreno J, Martinez-Aran A, Tabares-Seisdedos R, et al. Functioning and disability in bipolar disorder: an extensive review. Psychother. Psychosom 78 (2009): 285-297.

3. Akiskal HS, Benazzi F, Perugi G, Rihmer Z. Agitated "unipolar" depression reconceptualized as a depressive mixed state: implications for the antidepressant-suicide controversy. J Affect Disord 85 (2005): 245-258.

4. Akiskal HS, Bourgeois ML, Angst J, et al. Re-evaluating the prevalence of and diagnostic composition within the broad clinical spectrum of bipolar disorders. J Affect Disord 59 (2000): S5-S30.

5. González-Pinto A, Dardennes R, de Zélicourt M, et al. In-patient care costs of patients with bipolar I disorder: a comparison between two European centers. J Affect Disord 121 (2009): 152-155.

6. Kessler RC, Chiu WT, Demler O, et al. Corrections: Errors in Byline, Author Affiliations, and Acknowledgment in: Prevalence, Severity, and Comorbidity of 12-Month DSM-IV Disorders in the National Comorbidity Survey Replication. Archives of General Psychiatry 62 (2005): 709.

7. Merikangas KR, Ames M, Cui L, et al. The Impact of Comorbidity of Mental and Physical Conditions on Role Disability in the US Adult Household Population. Arch Gen Psychiatry 64 (2007): 1180-1188.

8. Miklowitz David J, Johnson Sheri L. The Psychopathology and Treatment of Bipolar Disorder. Annual Review of Clinical Psychology 2 (2006): 199-235.

9. Segal ZV, Williams JMG, Teasdale JD. Mindfulness-based cognitive therapy for depression: a new approach to preventing relapse. New York: Guilford Press (2002).

10. Williams JM, Alatiq Y, Crane C, et al. Mindfulness-based cognitive therapy (MBCT) in bipolar disorder: preliminary evaluation of immediate effects on between-episode functioning. J Affect Disord 107 (2008): 275-279.

11. Ma S, Teasdale JD. Mindfulness-based cognitive therapy for depression: replication and exploration of differential relapse prevention effects. J Consult Clin Psychol 72 (2004): 31-40.

12. Teasdale JD, Segal ZV, Williams JMG, et al. Prevention of relapse/recurrence in major depression by mindfulness-based cognitive therapy. J Consult Clin Psychol 68 (2000): 615-623.

13. Bondolfi G, Jermann F, der Linden MV, et al. Depression relapse prophylaxis with Mindfulness-based cognitive therapy: replication and extension in the Swiss health care system. J Affect Disord 122 (2010): 224-231. 
14. Kuyken W, Byford S, Taylor RS, et al. Mindfulness-based cognitive therapy to prevent relapse in recurrent depression. J Consult Clin Psychol 76 (2008): 966-978.

15. Eisendrath SJ, Delucchi K, Bitner R, et al. Mindfulness-based cognitive therapy for treatment-resistant depression: a pilot study. Psychother Psychosom 77 (2008): 319-320.

16. Finucane A, Mercer SW. An exploratory mixed methods study of the acceptability and effectiveness of Mindfulness-based cognitive therapy for patients with active depression and anxiety in primary care. BMC Psychiatry 6 (2006): 14.

17. Kingston T, Dooley B, Bates A, et al. Mindfulness-based cognitive therapy for residual depressive symptoms. Psychol Psychother 80 (2007): 193-203.

18. Kenny MA, Williams JMG. Treatment-resistant depressed patients show a good response to Mindfulnessbased cognitive therapy. Behav Res Ther 45 (2007): 617-625.

19. Miklowitz DJ, Alatiq Y, Goodwin GM, et al. A pilot study of mindfulness-based cognitive therapy for bipolar disorder. Int J Cogn Ther 2 (2009): 373-382.

20. Miklowitz DJ, George EL, Richards JA, et al. A randomized study of family focused psychoeducation and pharmacotherapy in the outpatient management of bipolar disorder. Arch Gen Psychiatry 60 (2003): 904912.

21. Reinares M, Vieta E, Colom F, et al. Impact of a psychoeducational family intervention on caregivers of stabilized bipolar patients. Psychother Psychosom 73 (2004): 312-319.

22. Jacobs LI. Cognitive therapy of postmanic and posted-pressive dysphoria in bipolar illness. Am J Psychother 36 (1982): 450-458.

23. Wehr TA. Can antidepressants induce rapid cycling? Arch Gen Psychiatry 50 (1993): 495-496.

24. Satterfield JM. Adjunctive cognitive-behavioral therapy for rapid-cycling bipolar disorder: an empirical case study. Psychiatry 62 (1999): 357-369.

25. Favre S, Aubry JM, Gex-Fabry M, et al. Traduction et validation franc, aise de l'e' chelle de manie de Young (YMRS). Encephale 29 (2003): 499-505.

26. Young RC, Biggs JT, Ziegler VE, et al. A rating scale for mania: reliability, validity and sensitivity. Br J Psychiatry 133 (1978): 429-435.

27. Montgomery SA, Asberg M. A new depression scale designed to be sensitive to change. Br J Psychiatry 134 (1979): 382-389.

Citation: Hajar Hasani ardekani, Hojaolah Javidi, Amir Hooshang Mehryar, Ebrahim Hoseni. Effectiveness of Integration of Mindfulness -Based Cognitive Therapy and Recovery Cognitive Behavioral Therapy on Adolescents with Spectrum Bipolar Disorder. Journal of Psychiatry and Psychiatric Disorders 2 (2018): 81-90.

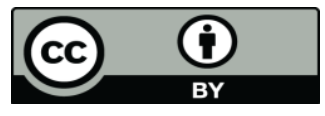

This article is an open access article distributed under the terms and conditions of the Creative Commons Attribution (CC-BY) license 4.0 